CRYSTALLOGRAPHIC

COMMUNICATIONS

ISSN 2056-9890

Reçu le 24 novembre 2014

Accepté le 26 novembre 2014

Édité par I. D. Brown, McMaster University, Canada

Keywords: crystal structure; triple molybdate; alluaudite-type

CCDC reference: 1036131

Supporting information: this article has supporting information at journals.iucr.org/e

\section{Structure cristalline de type alluaudite $\mathrm{K}_{0.4} \mathrm{Na}_{3.6} \mathrm{Co}\left(\mathrm{MoO}_{4}\right)_{3}$}

\author{
Rawia Nasri, Noura Fakhar Bourguiba* et Mohamed Faouzi Zid
}

Laboratoire de Matériaux et Cristallochimie, Faculté des Sciences de Tunis, Université de Tunis El Manar, 2092 El Manar Tunis, Tunisie. *Correspondence e-mail: n.f.bourguiba@live.fr

A new triple molybdate, potassium sodium cobalt tris(molybdate), $\mathrm{K}_{0.4} \mathrm{Na}_{3.6} \mathrm{Co}\left(\mathrm{MoO}_{4}\right)_{3}$, was synthesized using solid-state reactions. The $\mathrm{Co}^{2+}$ and one $\mathrm{Na}^{+}$cation are located at the same general site, each with occupancy 0.5 . Another site (site symmetry 2) is occupied by $\mathrm{Na}^{+}$and $\mathrm{K}^{+}$cations, with occupancies of 0.597 (7) and 0.402 (6), respectively. The other two $\mathrm{Na}^{+}$cations and one of the two Mo atoms lie on special positions (site symmetries $\overline{1}, 2$ and 2, respectively). The structure is characterized by $M_{2} \mathrm{O}_{10}(M=\mathrm{Co} / \mathrm{Na})$ dimers, which are linked by $\mathrm{MoO}_{4}$ tetrahedra, forming infinite layers. The latter are connected firstly by insertion of one type of $\mathrm{MoO}_{4}$ tetrahedra and secondly by sharing corners with the other type of $\mathrm{MoO}_{4}$ tetrahedra. This results in an open three-dimensional framework with the cavities occupied by the $\mathrm{Na}^{+}$and $\mathrm{K}^{+}$ cations. The structure is isotypic with $\mathrm{Na}_{3} \mathrm{In}_{2} \mathrm{As}_{3} \mathrm{O}_{12}$ and $\mathrm{Na}_{3} \mathrm{In}_{2} \mathrm{P}_{3} \mathrm{O}_{12}$. A comparison is made with structures such as $\mathrm{K}_{2} \mathrm{Co}_{2}\left(\mathrm{MoO}_{4}\right)_{3}$ and $\beta$ $\mathrm{NaFe}_{2}\left(\mathrm{MoO}_{4}\right)_{3}$ and their differences are discussed.

\section{Contexte chimique}

L'assemblage octaèdres-tétraèdres dans les matériaux inorganiques conduit à des charpentes ouvertes présentant des propriétés physiques importantes, en particulier la conduction ionique (Judeinstein et al., 1994; Sanz et al., 1999). La richesse structurale dans ces matériaux nous a encouragé de faire l'exploration des systèmes $A-\mathrm{Co}-\mathrm{Mo}-\mathrm{O}$ ( $A=$ ion monovalent). La synthèse conduit à un nouveau matériau de formulation $\mathrm{K}_{0.4} \mathrm{Na}_{3.6} \mathrm{Co}\left(\mathrm{MoO}_{4}\right)_{3}$ appartenant à la famille des alluaudites (Moore, 1971; Yakubovich et al., 2005; Hatert,

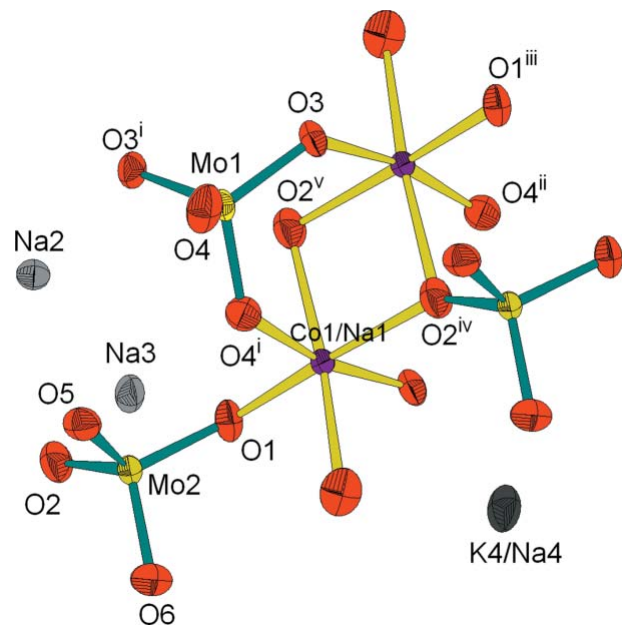

Figure 1

Unité asymétrique dans $\mathrm{K}_{0.4} \mathrm{Na}_{3.6} \mathrm{Co}\left(\mathrm{MoO}_{4}\right)_{3}$. Les éllipsoïdes ont été définis avec $50 \%$ de probabilité. [Code de symétrie: (i) $-x+1, y,-z+\frac{1}{2}$; (ii) $x,-y+1, z-\frac{1}{2}$; (iii) $-x+\frac{3}{2}, y-\frac{1}{2},-z+\frac{3}{2}$; (iv) $-x+\frac{3}{2},-y+\frac{1}{2},-z+1$; (v) $x,-y+1, z+\frac{1}{2}$. 


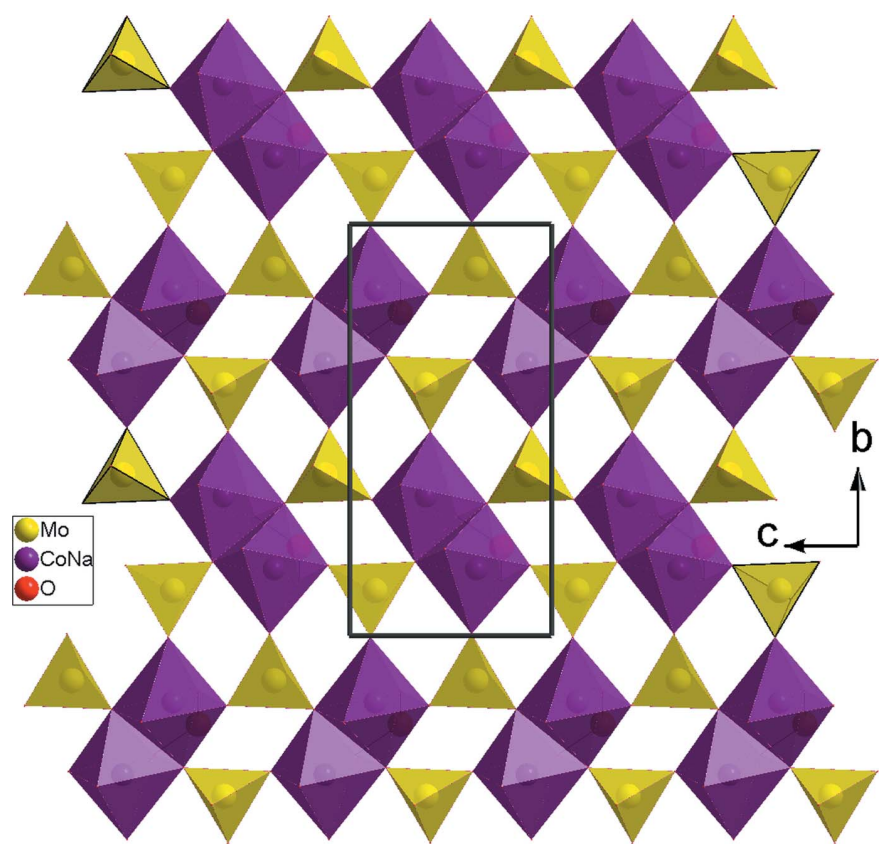

Figure 2

Projection d'une couche disposée parallèlement au plan (100).

2008). Un examen bibliographique montre que le matériau étudié est isostructural aux composés: $\mathrm{Na}_{3} \mathrm{In}_{2} \mathrm{As}_{3} \mathrm{O}_{12}$ et $\mathrm{Na}_{3} \mathrm{In}_{2} \mathrm{P}_{3} \mathrm{O}_{12}$ (Lii \& Ye, 1997).

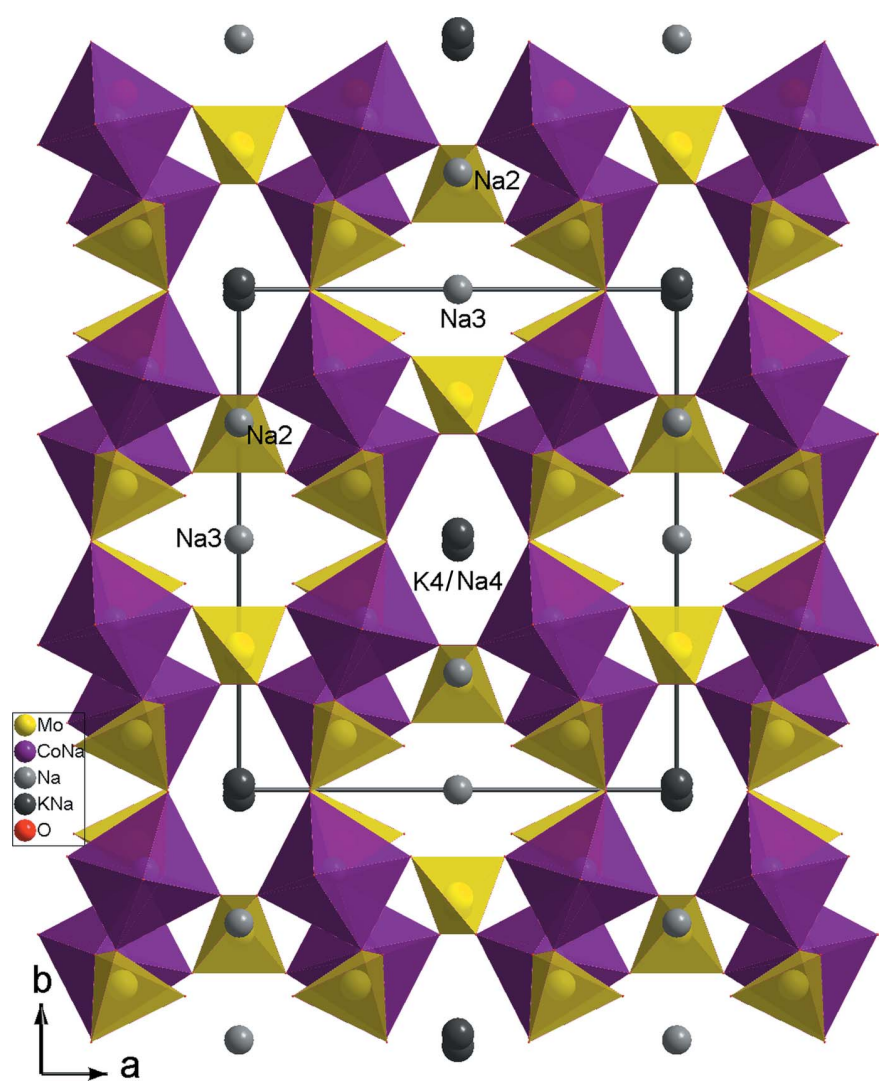

Figure 3

Projection de la structure de $\mathrm{K}_{0.4} \mathrm{Na}_{3.6} \mathrm{Co}\left(\mathrm{MoO}_{4}\right)_{3}$ selon $c$.

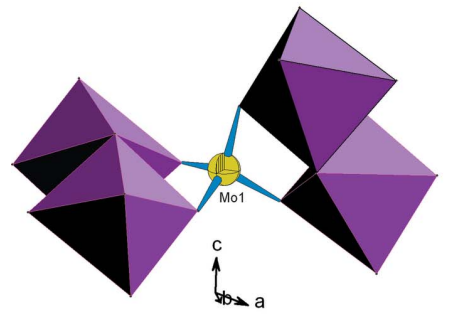

(a)

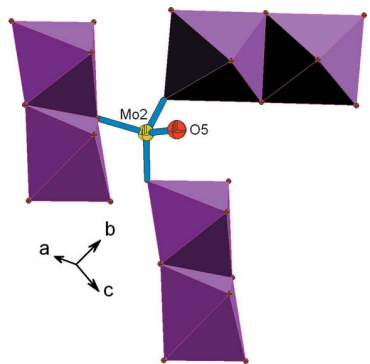

(b)
Figure 4

Représentation des environnements des tétraèdres: (a) $\mathrm{Mo1O}_{4},(b)$ $\mathrm{Mo}_{2} \mathrm{O}_{4}$.

\section{Commentaire structurelle}

L'unité asymétrique referme un dimère $M_{2} \mathrm{O}_{10}(M=\mathrm{Co} / \mathrm{Na})$ connecté par mise en commun de sommets avec deux tétraèdres et deux tétraèdres $\mathrm{Mo}_{2} \mathrm{O}_{4}$ différents et par partage d'arête avec un tétraèdres $\mathrm{Mo} \mathrm{O}_{4}$. La compensation de charge dans la structure est assurée par les cations $\mathrm{Na}^{+}$et $\mathrm{K}^{+}$(Fig. 1).

Dans la charpente anionique deux octaèdres juxtaposés, se lient par partage d'arête pour former des dimères $M_{2} \mathrm{O}_{10}(M=$ Co1/Na1). Ces derniers se connectent par ponts triples avec les tétraèdres $\mathrm{Mo}_{2} \mathrm{O}_{4}$ pour donner les unités $M_{2} \mathrm{Mo}_{2} \mathrm{O}_{16}$. Ces dernières se connectent à six autres unités identiques par mise en commun de sommets pour conduire à des couches infinies de type $M_{2} \mathrm{Mo}_{3} \mathrm{O}_{12}(M=\mathrm{Co} 1 / \mathrm{Na} 1)$ (Fig. 2).

Deux couches adjacentes se lient, d'une part par insertion des tétraèdres $\mathrm{Mo}_{4} \mathrm{O}_{4}$ entre les couches et d'autre part par partage de sommets pour conduire à une charpente tridimensionnelle, possédant deux types de canaux larges, à section hexagonale, parallèles à l'axe $c$ où logent les cations $\mathrm{Na} 3$ et (K4/Na4) (Fig. 3).

Dans la charpente anionique chaque tétraèdre $\mathrm{Mo1O}_{4}$ partage ses quatre sommets avec respectivement quatre octaèdres formant deux dimères appartenant à deux couches adjacentes (Fig. 4a). Par contre, dans la structure chaque tétraèdre $\mathrm{Mo}_{2} \mathrm{O}_{4}$ met en commun seulement trois de ses sommets avec respectivement trois dimères différents appartenant à la même couche, le quatrième sommet oxygène restant libre forme le groupement molybdyl $[d(M=\mathrm{O})=$ $1,744(2) \AA]$ (Fig. 4b). Un examen des caractéristiques géométriques relevées de l'étude structurale montre que les distances moyennes dans les tétraèdres $\mathrm{MoO}_{4}$ et dans les octaèdres $\mathrm{MO}_{6}(M=\mathrm{Co} 1 / \mathrm{Na} 1)$, sont égales respectivement à 1,761 (3) et 2,210 (3) $\AA$. La première Mo-O, est conforme à celles rencontrées dans la bibliographie (Souilem et al., 2014; Ennajeh et al., 2013; Engel et al., 2009). La seconde $M-\mathrm{O}$ (M $=\mathrm{Co} 1 / \mathrm{Na} 1)$, s'avère une moyenne entre celles $\mathrm{Co}-\mathrm{O}$ (Engel et al., 2009; Marzouki et al., 2013) et $\mathrm{Na}-\mathrm{O}$ (Muessig et al., 2003; Baies et al., 2006). De plus, le calcul des charges des ions, utilisant la formule empirique de Brown (Brown \& Altermatt, 1985), conduit aux valeurs des charges des ions suivants: Mo1 $(5,943), \mathrm{Mo} 2(5,946), \mathrm{Co} 1 \mathrm{Na} 1(1,822), \mathrm{Na} 2(1,093), \mathrm{Na} 3$ $(0,838), \mathrm{K} 4 / \mathrm{Na} 4(0,868)$. La structure étudiée étant de type alluaudite, elle est similaire à celles rencontrées dans la 


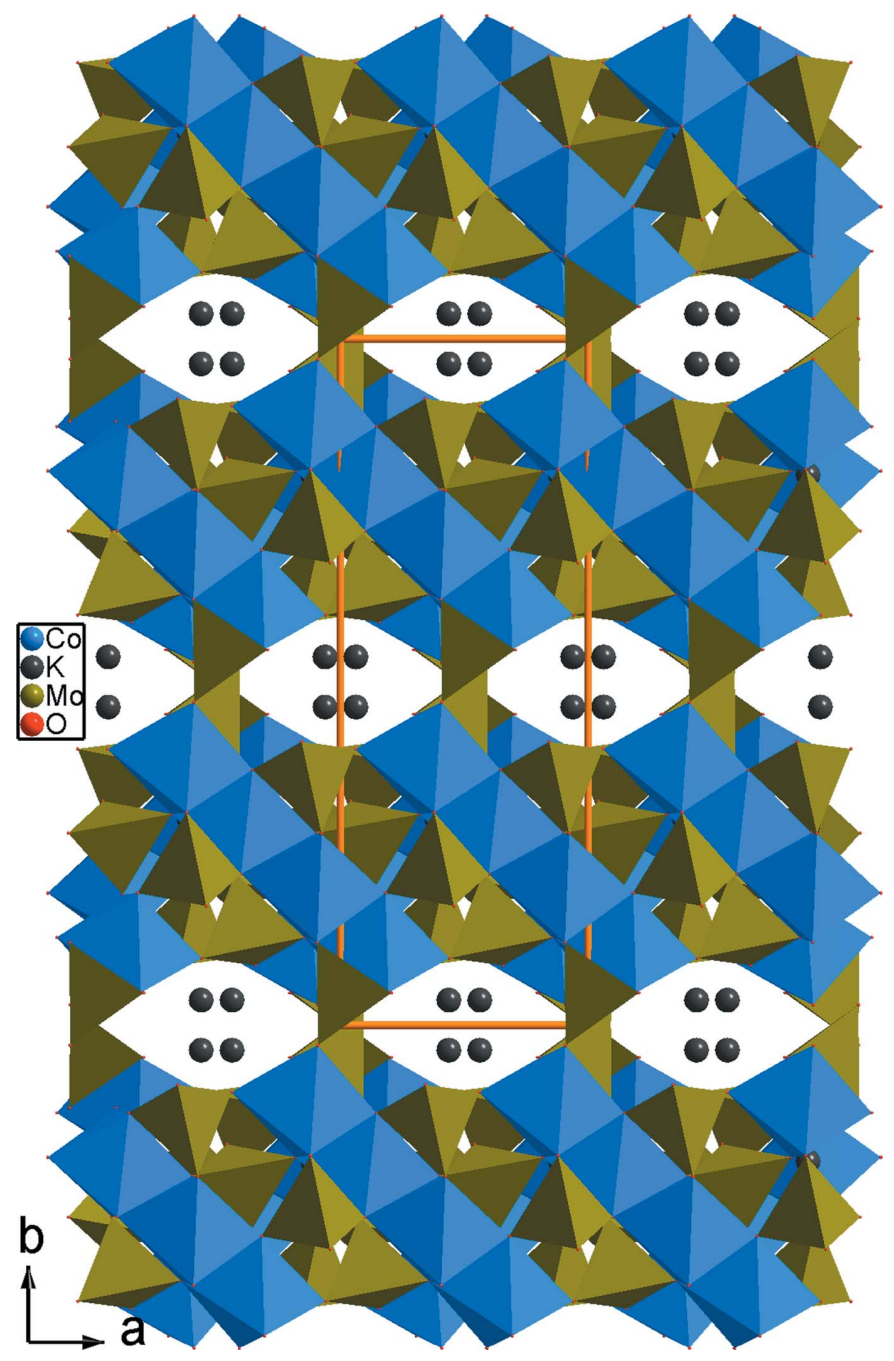

Figure 5

Projection de la structure de $\mathrm{K}_{2} \mathrm{Co}_{2}\left(\mathrm{MoO}_{4}\right)_{3}$, selon $c$.

littérature (Haj Abdallah \& Haddad, 2008; Zid et al., 2005). Elles diffèrent seulement par l'occupation des sites cationiques. En effet, dans le composé $\mathrm{Na}_{1.72} \mathrm{Mn}_{3.28}\left(\mathrm{AsO}_{4}\right)_{3}$ (Ayed et al., 2002), le site cristallographique $(1 / 2, y, 3 / 4) \mathrm{du}$ groupe d'espace $C 2 / c$, est occupé par l'atome de manganèse $\mathrm{Mn} 1$, contrairement à notre phase où ce site est occupé par l'alcalin $\mathrm{Na} 2$ (Fig. 3).

\section{Enquête de base de données}

Le composé de formulation $\mathrm{K}_{2} \mathrm{Co}_{2}\left(\mathrm{MoO}_{4}\right)_{3}$ (Engel et al., 2009), présente trois variétés allotropiques. Elles cristallisent dans le systéme monoclinique, groupes d'espace $P 2_{1} / c$ ou bien $C 2 / c$. La charpente anionique dans $\mathrm{K}_{2} \mathrm{Co}_{2}\left(\mathrm{MoO}_{4}\right)_{3}$ présente contrairement à notre structure des tétramères (Fig. 5) au lieu des dimères. L'association, par partage de sommets, des tétramères avec tous les tétraèdres $\mathrm{MoO}_{4}$ dans $\mathrm{K}_{2} \mathrm{Co}_{2}\left(\mathrm{MoO}_{4}\right)_{3}$ conduit vers une structure bidimensionnelle. La comparaison de notre structure avec celle de la variété $\beta$ - $\mathrm{NaFe}_{2}\left(\mathrm{MoO}_{4}\right)_{3}$ (Muessig et al., 2003), montre une différence dans la disposi-

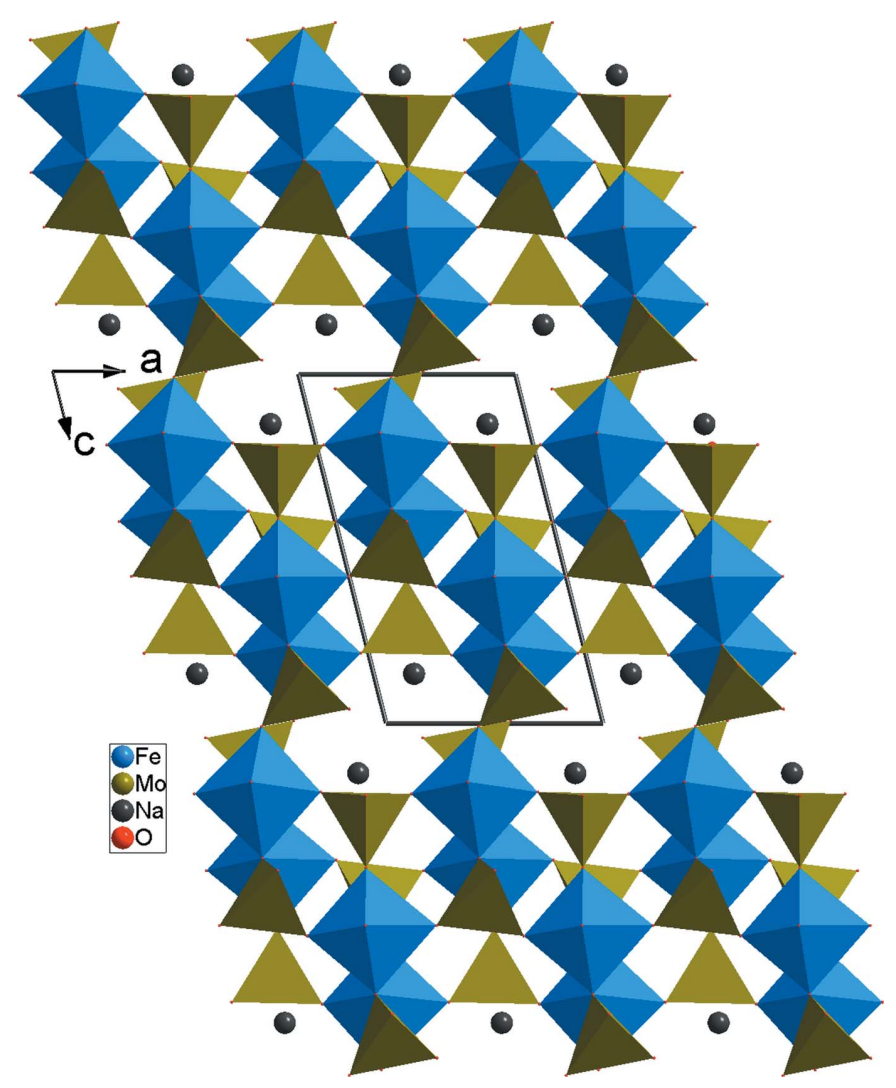

Figure 6

Projection de la structure de la variété $\beta-\mathrm{NaFe}_{2}\left(\mathrm{MoO}_{4}\right)_{3}$, selon $b$.

tion des dimères $M_{2} \mathrm{O}_{10}(M=\mathrm{Co} / \mathrm{Fe})$. En effet dans la structure $\beta$ - $\mathrm{NaFe}_{2}\left(\mathrm{MoO}_{4}\right)_{3}$ les dimères sont orientés de la même façon. Leur association par partage de sommets avec les tétraèdres $\mathrm{MoO}_{4}$ conduit vers une charpente ouverte possédant de larges canaux où résident les cations $\mathrm{Na}^{+}$(Fig. 6). Contrairement à notre matériau $\mathrm{K}_{0.4} \mathrm{Na}_{3.6} \mathrm{Co}\left(\mathrm{MoO}_{4}\right)_{3}$ où les dimères $M_{2} \mathrm{O}_{10}(M=\mathrm{Co} / \mathrm{Na})$ sont orientés perpendiculairement les uns aux autres (Fig. 3).

\section{Synthèse et cristallisation}

Les cristaux relatifs à $\mathrm{K}_{0.4} \mathrm{Na}_{3.6} \mathrm{Co}\left(\mathrm{MoO}_{4}\right)_{3}$ ont été obtenus par réaction à l'état solide à partir des réactifs: $\mathrm{Na}_{2} \mathrm{CO}_{3}$ (PROLABO, 70128), $\mathrm{Co}\left(\mathrm{NO}_{3}\right) \cdot 6 \mathrm{H}_{2} \mathrm{O}$ (FLUKA, 60832), $\mathrm{K}_{2} \mathrm{CO}_{3}$ (PROLABO, 60109) et $\left(\mathrm{NH}_{4}\right)_{2} \mathrm{Mo}_{4} \mathrm{O}_{13}$ (FLUKA, 69858) pris dans les proportions telque les rapports $\mathrm{Na}: \mathrm{K}: \mathrm{Co}: \mathrm{Mo}=2: 1: 3: 3$. Après un broyage poussé dans un mortier en agate, le mélange a été mis dans un creuset en porcelaine préchauffé à l'air à $673 \mathrm{~K}$ pendant 12 heures en vue d'éliminer les composés volatils. Il est ensuite porté jusqu'à une température de synthèse proche de celle de la fusion à $963 \mathrm{~K}$. Le mélange est maintenue à cette température pendant deux semaines pour favoriser la germination et la croissance des cristaux. Par la suite, il a subi en premier lieu un refroidissement lent (5\%/jour) jusqu'à $910 \mathrm{~K}$ puis rapide $\left(50^{\circ} / \mathrm{h}\right)$ jusqu'à la température ambiante. Des cristaux de couleur violet, de taille suffisante pour les mesures des 


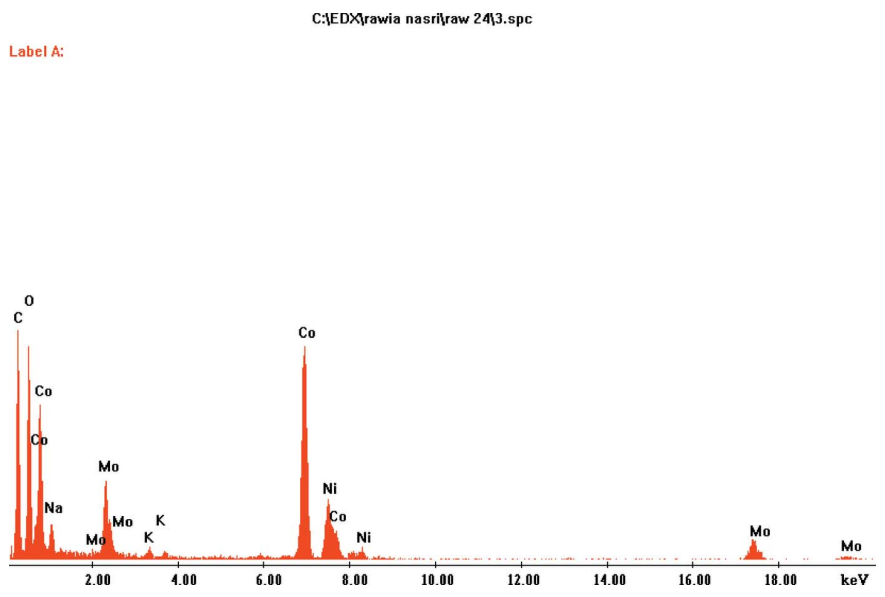

Figure 7

Analyse MET d'un cristal choisi de $\mathrm{K}_{0.4} \mathrm{Na}_{3.6} \mathrm{Co}\left(\mathrm{MoO}_{4}\right)_{3}$.

intensités, ont été séparés du flux par l'eau chaude. Une analyse qualitative au MET de marque FEI et de type QUANTA 200 confirme la présence des éléments chimiques attendus: K, Na, Co, Mo et l'oxygène (Fig. 7).

\section{Affinement}

Détails de données crystallines, collection de données et affinement sont résumées dans le tableau 1. Des contraintes EADP et EXYZ pour les couples $\mathrm{K} 4 / \mathrm{Na} 4$ conduit à des ellipsoïdes bien définis. Les densités d'électrons residuelles sont acceptables et sont situées respectivements à $0,80 \AA$ de Mo2 et à $0,99 \AA$ Ad de Mo1.

\section{Références}

Ayed, B., Krifa, M. \& Haddad, A. (2002). Acta Cryst. C58, i98-i100. Baies, R., Pérez, O., Caignaert, V., Pralong, V. \& Raveau, B. (2006). J. Mater. Chem. 16, 2434-2438.

Brandenburg, K. \& Putz, H. (1999). DIAMOND. Crystal Impact GbR, Bonn, Allemagne.

Brown, I. D. \& Altermatt, D. (1985). Acta Cryst. B41, 244-247.

Duisenberg, A. J. M. (1992). J. Appl. Cryst. 25, 92-96.

Engel, J. M., Ahsbahs, H., Fuess, H. \& Ehrenberg, H. (2009). Acta Cryst. B65, 29-35.

Ennajeh, I., Zid, M. F. \& Driss, A. (2013). Acta Cryst. E69, i54-i55. Farrugia, L. J. (2012). J. Appl. Cryst. 45, 849-854.

Haj Abdallah, A. \& Haddad, A. (2008). Acta Cryst. E64, i36.

Harms, K. \& Wocadlo, S. (1995). XCAD4. University of Marburg, Allemagne.
Tableau 1

Détails expérimentaux.

\begin{tabular}{|c|c|}
\hline \multicolumn{2}{|l|}{ Données crystallines } \\
\hline Formule chimique & $\mathrm{K}_{0.4} \mathrm{Na}_{3.6} \mathrm{Co}\left(\mathrm{MoO}_{4}\right)_{3}$ \\
\hline$M_{\mathrm{r}}$ & 637,15 \\
\hline Système cristallin, groupe d'espace & Monoclinique, $C 2 / c$ \\
\hline Température (K) & 298 \\
\hline$a, b, c(\AA)$ & $12,8054(8), 13,5328(9), 7,1888(6)$ \\
\hline$\beta\left({ }^{\circ}\right)$ & $112,239(8)$ \\
\hline$V\left(\AA^{3}\right)$ & $1153,10(14)$ \\
\hline$Z$ & 4 \\
\hline Type de rayonnement & Mo $K \alpha$ \\
\hline$\mu\left(\mathrm{mm}^{-1}\right)$ & 4.94 \\
\hline Taille des cristaux (mm) & $0,22 \times 0,16 \times 0,12$ \\
\hline \multicolumn{2}{|l|}{ Collection de données } \\
\hline Diffractomètre & Enraf-Nonius CAD-4 \\
\hline Correction d'absorption & $\psi$ scan (North et al., 1968) \\
\hline$T_{\min }, T_{\max }$ & $0,557,0,607$ \\
\hline $\begin{array}{l}\text { Nombre de réflexions mesurées, } \\
\text { indépendantes et observées } \\
{[I>2 \sigma(I)]}\end{array}$ & $2813,1252,1092$ \\
\hline$R_{\mathrm{int}}$ & 0,029 \\
\hline$(\sin \theta / \lambda)_{\max }\left(\AA^{-1}\right)$ & 0,638 \\
\hline \multicolumn{2}{|l|}{ Affinement } \\
\hline$R\left[F^{2}>2 \sigma\left(F^{2}\right)\right], w R\left(F^{2}\right), S$ & $0,022,0,056,1,05$ \\
\hline Nombre de réflexions & 1252 \\
\hline Nombre de paramètres & 99 \\
\hline Nombre de restraints & 1 \\
\hline$\Delta \rho_{\max }, \Delta \rho_{\min }\left(\mathrm{e} \AA^{-3}\right)$ & $0,47,-0,43$ \\
\hline
\end{tabular}

Programmes informatiques: CAD-4 EXPRESS (Duisenberg, 1992; Macíček \& Yordanov, 1992), XCAD4 (Harms \& Wocadlo, 1995), SHELXS97 et SHELXL97 (Sheldrick, 2008), DIAMOND (Brandenburg \& Putz, 1999) et WinGX (Farrugia, 2012).

Hatert, F. (2008). J. Solid State Chem. 181, 1258-1272.

Judeinstein, P., Titman, J., Stamm, M. \& Schmidt, H. (1994). Chem. Mater. 6, 127-134.

Lii, K.-H. \& Ye, J. (1997). J. Solid State Chem. 131, 131-137.

Macíček, J. \& Yordanov, A. (1992). J. Appl. Cryst. 25, 73-80.

Marzouki, R., Frigui, W., Guesmi, A., Zid, M. F. \& Driss, A. (2013). Acta Cryst. E69, i65-i66.

Moore, P. B. (1971). Am. Mineral. 56, 1955-1975.

Muessig, E., Bramnik, K. G. \& Ehrenberg, H. (2003). Acta Cryst. B59, 611-616.

North, A. C. T., Phillips, D. C. \& Mathews, F. S. (1968). Acta Cryst. A24, 351-359.

Sanz, F., Parada, C., Rojo, J. M., Ruiz-Valero, C. \& Saez-Puche, R. (1999). J. Solid State Chem. 145, 604-611.

Sheldrick, G. M. (2008). Acta Cryst. A64, 112-122.

Souilem, A., Zid, M. F. \& Driss, A. (2014). Acta Cryst. E70, i9-i10.

Yakubovich, O. V., Massa, W., Gavrilenko, P. G. \& Dimitrova, O. V. (2005). Eur. J. Mineral. 17, 741-748.

Zid, M. F., Driss, A. \& Jouini, T. (2005). Acta Cryst. E61, i46-i48. 


\section{supporting information}

Acta Cryst. (2015). E71, 4-7 [https://doi.org/10.1107/S2056989014025894]

\section{Structure cristalline de type alluaudite $\mathrm{K}_{0.4} \mathrm{Na}_{3.6} \mathrm{Co}\left(\mathrm{MoO}_{4}\right)_{3}$}

\section{Rawia Nasri, Noura Fakhar Bourguiba et Mohamed Faouzi Zid}

\section{Computing details}

Data collection: CAD-4 EXPRESS (Duisenberg, 1992; Macíček \& Yordanov, 1992); cell refinement: CAD-4 EXPRESS (Duisenberg, 1992; Macíček \& Yordanov, 1992); data reduction: XCAD4 (Harms \& Wocadlo, 1995); program(s) used to solve structure: SHELXS97 (Sheldrick, 2008); program(s) used to refine structure: SHELXL97 (Sheldrick, 2008); molecular graphics: DIAMOND (Brandenburg \& Putz, 1999); software used to prepare material for publication: WinGX (Farrugia, 2012).

Potassium sodium cobalt tris(molybdate)

Crystal data

$\mathrm{K}_{0.4} \mathrm{Na}_{3.6} \mathrm{Co}\left(\mathrm{MoO}_{4}\right)_{3}$

$M_{r}=637.15$

Monoclinic, $C 2 / c$

Hall symbol: $-\mathrm{C} 2 \mathrm{yc}$

$a=12.8054(8) \AA$

$b=13.5328(9) \AA$

$c=7.1888(6) \AA$

$\beta=112.239(8)^{\circ}$

$V=1153.10(14) \AA^{3}$

$Z=4$

\section{Data collection}

Enraf-Nonius CAD-4 diffractometer

Radiation source: fine-focus sealed tube Graphite monochromator $\omega / 2 \theta$ scans

Absorption correction: $\psi$ scan

(North et al., 1968)

$T_{\min }=0.557, T_{\max }=0.607$

2813 measured reflections

\section{Refinement}

Refinement on $F^{2}$

Least-squares matrix: full

$R\left[F^{2}>2 \sigma\left(F^{2}\right)\right]=0.022$

$w R\left(F^{2}\right)=0.056$

$S=1.05$

1252 reflections

99 parameters

1 restraint
$F(000)=1185$

$D_{\mathrm{x}}=3.670 \mathrm{Mg} \mathrm{m}^{-3}$

Mo $K \alpha$ radiation, $\lambda=0.71073 \AA$

Cell parameters from 25 reflections

$\theta=10-15^{\circ}$

$\mu=4.94 \mathrm{~mm}^{-1}$

$T=298 \mathrm{~K}$

Prism, purple

$0.22 \times 0.16 \times 0.12 \mathrm{~mm}$

1252 independent reflections

1092 reflections with $I>2 \sigma(I)$

$R_{\text {int }}=0.029$

$\theta_{\max }=27.0^{\circ}, \theta_{\min }=2.3^{\circ}$

$h=-16 \rightarrow 13$

$k=-2 \rightarrow 17$

$l=-9 \rightarrow 9$

2 standard reflections every $120 \mathrm{~min}$ intensity decay: $1.4 \%$

Primary atom site location: structure-invariant direct methods

Secondary atom site location: difference Fourier map

$w=1 /\left[\sigma^{2}\left(F_{\mathrm{o}}^{2}\right)+(0.0256 P)^{2}+0.2937 P\right]$

where $P=\left(F_{\mathrm{o}}{ }^{2}+2 F_{\mathrm{c}}{ }^{2}\right) / 3$

$(\Delta / \sigma)_{\max }<0.001$

$\Delta \rho_{\max }=0.47 \mathrm{e} \AA^{-3}$ 
$\Delta \rho_{\min }=-0.43$ e $\AA^{-3}$
Extinction correction: SHELXL97 (Sheldrick, 2008), $\mathrm{Fc}^{*}=\mathrm{kFc}\left[1+0.001 \times \mathrm{xc}^{2} \lambda^{3} / \sin (2 \theta)\right]^{-1 / 4}$

Extinction coefficient: 0.00215 (15)

Special details

Geometry. All e.s.d.'s (except the e.s.d. in the dihedral angle between two 1.s. planes) are estimated using the full covariance matrix. The cell e.s.d.'s are taken into account individually in the estimation of e.s.d.'s in distances, angles and torsion angles; correlations between e.s.d.'s in cell parameters are only used when they are defined by crystal symmetry. An approximate (isotropic) treatment of cell e.s.d.'s is used for estimating e.s.d.'s involving 1.s. planes.

Refinement. Refinement of $F^{2}$ against ALL reflections. The weighted $R$-factor $w R$ and goodness of fit $S$ are based on $F^{2}$, conventional $R$-factors $R$ are based on $F$, with $F$ set to zero for negative $F^{2}$. The threshold expression of $F^{2}>\sigma\left(F^{2}\right)$ is used only for calculating $R$-factors(gt) etc. and is not relevant to the choice of reflections for refinement. $R$-factors based on $F^{2}$ are statistically about twice as large as those based on $F$, and $R$ - factors based on ALL data will be even larger.

Fractional atomic coordinates and isotropic or equivalent isotropic displacement parameters $\left(\AA^{2}\right)$

\begin{tabular}{llllll}
\hline & $x$ & $y$ & $z$ & $U_{\text {iso }} * / U_{\text {eq }}$ & Occ. $(<1)$ \\
\hline Mo1 & 0.5000 & $0.21535(3)$ & 0.2500 & $0.02386(14)$ & \\
Mo2 & $0.73366(2)$ & $0.60949(2)$ & $0.62347(4)$ & $0.02137(12)$ & \\
Co1 & $0.71350(6)$ & $0.33825(6)$ & $0.62440(10)$ & $0.01883(17)$ & 0.50 \\
Na1 & $0.71350(6)$ & $0.33825(6)$ & $0.62440(10)$ & $0.01883(17)$ & 0.50 \\
Na2 & 0.5000 & $0.23393(16)$ & 0.7500 & $0.0245(4)$ & \\
Na3 & 0.5000 & 0.0000 & 0.5000 & $0.0358(5)$ & \\
K4 & 0.5000 & $0.48817(18)$ & 0.2500 & $0.0434(8)$ & $0.402(16)$ \\
Na4 & 0.5000 & $0.48817(18)$ & 0.2500 & $0.0434(8)$ & $0.597(18)$ \\
O1 & $0.6713(2)$ & $0.6703(2)$ & $0.3919(4)$ & $0.0310(6)$ & \\
O2 & $0.7192(2)$ & $0.6791(2)$ & $0.8226(4)$ & $0.0367(7)$ & \\
O3 & $0.5413(2)$ & $0.2891(2)$ & $0.4691(4)$ & $0.0291(6)$ & \\
O4 & $0.6072(3)$ & $0.1336(2)$ & $0.2506(5)$ & $0.0461(8)$ & \\
O5 & $0.8768(2)$ & $0.5878(2)$ & $0.6818(4)$ & $0.0329(6)$ & \\
O6 & $0.6623(3)$ & $0.4960(2)$ & $0.6026(5)$ & $0.0438(8)$ & \\
\end{tabular}

Atomic displacement parameters $\left(\AA^{2}\right)$

\begin{tabular}{lllllll}
\hline & $U^{11}$ & $U^{22}$ & $U^{33}$ & $U^{12}$ & $U^{13}$ & $U^{23}$ \\
\hline Mo1 & $0.0371(3)$ & $0.0170(2)$ & $0.0127(2)$ & 0.000 & $0.00406(17)$ & 0.000 \\
Mo2 & $0.02105(18)$ & $0.0256(2)$ & $0.01562(17)$ & $-0.00171(12)$ & $0.00482(12)$ & $0.00182(11)$ \\
Co1 & $0.0207(3)$ & $0.0214(4)$ & $0.0145(3)$ & $0.0035(3)$ & $0.0068(3)$ & $0.0003(3)$ \\
Na1 & $0.0207(3)$ & $0.0214(4)$ & $0.0145(3)$ & $0.0035(3)$ & $0.0068(3)$ & $0.0003(3)$ \\
Na2 & $0.0237(9)$ & $0.0250(11)$ & $0.0276(10)$ & 0.000 & $0.0129(8)$ & 0.000 \\
Na3 & $0.0478(14)$ & $0.0220(11)$ & $0.0246(11)$ & $-0.0008(11)$ & $-0.0011(10)$ & $0.0008(9)$ \\
K4 & $0.0238(10)$ & $0.0710(17)$ & $0.0308(10)$ & 0.000 & $0.0051(7)$ & 0.000 \\
Na4 & $0.0238(10)$ & $0.0710(17)$ & $0.0308(10)$ & 0.000 & $0.0051(7)$ & 0.000 \\
O1 & $0.0330(14)$ & $0.0347(16)$ & $0.0204(12)$ & $0.0102(13)$ & $0.0046(11)$ & $0.0015(12)$ \\
O2 & $0.0410(16)$ & $0.0462(18)$ & $0.0242(14)$ & $0.0009(14)$ & $0.0139(12)$ & $0.0005(13)$ \\
O3 & $0.0328(13)$ & $0.0357(15)$ & $0.0189(12)$ & $-0.0025(12)$ & $0.0098(10)$ & $-0.0051(11)$ \\
O4 & $0.058(2)$ & $0.0285(16)$ & $0.0375(16)$ & $0.0091(15)$ & $0.0012(15)$ & $-0.0093(14)$ \\
O5 & $0.0270(14)$ & $0.0289(15)$ & $0.0400(16)$ & $0.0025(12)$ & $0.0096(12)$ & $0.0084(13)$ \\
O6 & $0.0439(18)$ & $0.0396(18)$ & $0.0457(18)$ & $-0.0114(15)$ & $0.0145(14)$ & $0.0076(15)$ \\
& & & & & & \\
\hline
\end{tabular}


Geometric parameters $\left(\AA,{ }^{o}\right)$

\begin{tabular}{|c|c|c|c|}
\hline $\mathrm{Mo} 1-\mathrm{O} 4^{\mathrm{i}}$ & $1.762(3)$ & $\mathrm{Na} 2-\mathrm{O} 1^{\mathrm{vii}}$ & $2.416(3)$ \\
\hline Mo1-O4 & $1.762(3)$ & $\mathrm{Na} 2-\mathrm{O}^{\text {viii }}$ & $2.461(3)$ \\
\hline $\mathrm{Mo} 1-\mathrm{O} 3$ & $1.768(2)$ & $\mathrm{Na} 2-\mathrm{O}^{\mathrm{v}}$ & $2.461(3)$ \\
\hline $\mathrm{Mo1}-\mathrm{O}^{\mathrm{i}}$ & $1.768(2)$ & $\mathrm{Na} 3-\mathrm{O}^{\text {ix }}$ & $2.530(3)$ \\
\hline Mo2-O5 & $1.744(3)$ & $\mathrm{Na} 3-\mathrm{O}^{\mathrm{v}}$ & $2.530(3)$ \\
\hline Mo2-O1 & $1.755(3)$ & $\mathrm{Na} 3-\mathrm{O} 4^{\mathrm{i}}$ & $2.554(3)$ \\
\hline Mo2-O6 & $1.764(3)$ & $\mathrm{Na} 3-\mathrm{O} 4^{\mathrm{x}}$ & $2.554(3)$ \\
\hline $\mathrm{Mo} 2-\mathrm{O} 2$ & $1.781(3)$ & $\mathrm{Na} 3-\mathrm{O} 5^{\mathrm{ii}}$ & $2.677(3)$ \\
\hline $\mathrm{Co} 1-\mathrm{O} 4^{\mathrm{ii}}$ & $2.159(4)$ & $\mathrm{Na} 3-\mathrm{O}^{\text {viii }}$ & $2.677(3)$ \\
\hline $\mathrm{Co} 1-\mathrm{O} 3$ & $2.166(3)$ & $\mathrm{K} 4-\mathrm{O} 6$ & $2.602(3)$ \\
\hline $\mathrm{Co} 1-\mathrm{O} 1^{\mathrm{iii}}$ & $2.188(3)$ & $\mathrm{K} 4-\mathrm{O}^{\mathrm{i}}$ & $2.602(3)$ \\
\hline $\mathrm{Co} 1-\mathrm{O} 2^{\mathrm{iv}}$ & $2.211(3)$ & $\mathrm{K} 4-\mathrm{O}^{\mathrm{iv}}$ & $2.675(3)$ \\
\hline $\mathrm{Co} 1-\mathrm{O} 6$ & $2.222(3)$ & $\mathrm{K} 4-\mathrm{O}^{\mathrm{vii}}$ & $2.675(3)$ \\
\hline $\mathrm{Co} 1-\mathrm{O} 2^{\mathrm{v}}$ & $2.298(3)$ & $\mathrm{K} 4-\mathrm{O} 3^{\mathrm{i}}$ & $3.064(3)$ \\
\hline $\mathrm{Na} 2-\mathrm{O}^{\mathrm{vi}}$ & $2.392(3)$ & $\mathrm{K} 4-\mathrm{O} 3$ & $3.064(3)$ \\
\hline $\mathrm{Na} 2-\mathrm{O} 3$ & $2.392(3)$ & $\mathrm{K} 4-\mathrm{O} 1$ & $3.199(4)$ \\
\hline $\mathrm{Na} 2-\mathrm{O} 1^{\mathrm{iii}}$ & $2.416(3)$ & $\mathrm{K} 4-\mathrm{O} 1^{\mathrm{i}}$ & $3.199(4)$ \\
\hline $\mathrm{O} 4-\mathrm{Mo} 1-\mathrm{O} 4$ & $102.2(2)$ & $\mathrm{O} 3-\mathrm{Co} 1-\mathrm{O} 1^{\mathrm{iii}}$ & $83.96(10)$ \\
\hline $\mathrm{O} 4 \mathrm{i}-\mathrm{Mo} 1-\mathrm{O} 3$ & $109.06(14)$ & $\mathrm{O} 4^{\mathrm{ii}}-\mathrm{Co} 1-\mathrm{O} 2^{\mathrm{iv}}$ & $90.15(12)$ \\
\hline $\mathrm{O} 4-\mathrm{Mo1}-\mathrm{O} 3$ & $112.50(13)$ & $\mathrm{O} 3-\mathrm{Co} 1-\mathrm{O} 2^{\mathrm{iv}}$ & $82.34(11)$ \\
\hline $\mathrm{O} 4^{\mathrm{i}}-\mathrm{Mo} 1-\mathrm{O}^{\mathrm{i}}$ & $112.50(13)$ & 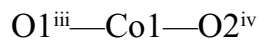 & $165.35(11)$ \\
\hline $\mathrm{O} 4-\mathrm{Mo} 1-\mathrm{O}^{\mathrm{i}}$ & $109.06(14)$ & $\mathrm{O} 4^{\mathrm{ii}}-\mathrm{Co} 1-\mathrm{O} 6$ & $95.65(12)$ \\
\hline $\mathrm{O} 3-\mathrm{Mo} 1-\mathrm{O}^{\mathrm{i}}$ & $111.26(18)$ & $\mathrm{O} 3-\mathrm{Co} 1-\mathrm{O} 6$ & $92.46(11)$ \\
\hline $\mathrm{O} 5-\mathrm{Mo} 2-\mathrm{O} 1$ & $111.51(13)$ & $\mathrm{O} 1 \mathrm{iii}-\mathrm{Co} 1-\mathrm{O} 6$ & 87.03 (12) \\
\hline $\mathrm{O} 5-\mathrm{Mo} 2-\mathrm{O} 6$ & $109.70(15)$ & $\mathrm{O} 2^{\mathrm{iv}}-\mathrm{Co} 1-\mathrm{O} 6$ & $98.70(12)$ \\
\hline $\mathrm{O} 1-\mathrm{Mo} 2-\mathrm{O} 6$ & $107.00(15)$ & $\mathrm{O} 4^{\mathrm{ii}}-\mathrm{Co} 1-\mathrm{O} 2^{\mathrm{v}}$ & $79.83(11)$ \\
\hline $\mathrm{O} 5-\mathrm{Mo} 2-\mathrm{O} 2$ & $108.41(14)$ & $\mathrm{O} 3-\mathrm{Co} 1-\mathrm{O} 2^{\mathrm{v}}$ & $92.45(11)$ \\
\hline $\mathrm{O} 1-\mathrm{Mo} 2-\mathrm{O} 2$ & $111.48(13)$ & $\mathrm{O} 1^{\mathrm{iii}}-\mathrm{Co} 1-\mathrm{O} 2^{\mathrm{v}}$ & $90.38(11)$ \\
\hline $\mathrm{O} 6-\mathrm{Mo} 2-\mathrm{O} 2$ & $108.69(15)$ & $\mathrm{O} 2^{\mathrm{iv}}-\mathrm{Co} 1-\mathrm{O} 2^{\mathrm{v}}$ & $85.05(11)$ \\
\hline $\mathrm{O} 44^{\mathrm{ii}}-\mathrm{Co} 1-\mathrm{O} 3$ & $169.69(11)$ & $\mathrm{O} 6-\mathrm{Co} 1-\mathrm{O}^{\mathrm{v}}$ & $174.18(11)$ \\
\hline 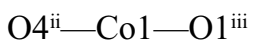 & $102.76(12)$ & & \\
\hline
\end{tabular}

Symmetry codes: (i) $-x+1, y,-z+1 / 2$; (ii) $-x+3 / 2,-y+1 / 2,-z+1$; (iii) $x,-y+1, z+1 / 2$; (iv) $x,-y+1, z-1 / 2$; (v) $-x+3 / 2, y-1 / 2,-z+3 / 2$; (vi) $-x+1, y,-z+3 / 2$; (vii) $-x+1,-y+1,-z+1$; (viii) $x-1 / 2, y-1 / 2, z$; (ix) $x-1 / 2,-y+1 / 2, z-1 / 2$; (x) $x,-y, z+1 / 2$. 